7th International Workshop on Astronomy and

Relativistic Astrophysics (IWARA 2016)

International Journal of Modern Physics: Conference Series

Vol. 45 (2017) 1760042 (6 pages)

(C) The Author(s)

DOI: $10.1142 / S 2010194517600424$

\title{
Nucleosynthesis in Strange Star Mergers
}

\author{
Laura Paulucci \\ Universidade Federal do $A B C$ (UFABC) \\ Av. dos Estados, 5001 \\ 09210-170 Santo André, SP, Brazil \\ laura.paulucci@ufabc.edu.br \\ Jorge E. Horvath \\ Instituto de Astronomia, Geofísica e Ciências Atmosféricas (IAG) \\ Universidade de São Paulo (USP) \\ R. do Matão, 1226 \\ São Paulo, SP, 05508-090, Brazil \\ foton@iag.usp.br \\ Omar Benvenuto \\ Facultad de Ciencias Astronômicas y Geofísicas \\ Universidad Nacional de La Plata (UNLP) \\ Paseo del Bosque $S / N$ \\ B1900FWA La Plata, Argentina \\ obenvenu@fcaglp.unlp.edu.ar
}

Published 15 August 2017

The possible existence of deconfined matter in the cores of neutron stars has been studied for over three decades without a firm indication either for or against this proposition. Analysis mostly rely on the comparison of mass-radius curves obtained for different compositions with observational data on the mass of the most massive objects of this kind accurately determined. Nevertheless, there are other possibilities for indirectly studying the internal composition of this class of compact objects, e.g, analyzing cooling behavior, X-ray bursts, supernova's neutrinos. We present calculations on the expected nucleosynthesis spectra for the strange star-strange star merger scenario as means to test the strange quark matter hypothesis and its realization inside such objects. This would result very different from the typical r-process nucleosynthesis expected in neutron star mergers since the high temperature deconfinement of strange matter would produce large amounts of neutrons and protons and the mass buildup would proceed in a Big-Bang nucleosynthesis like scenario. The neutron to proton ratio would allow to reach the iron peak only, a very different prediction from the standard scenario. The resultant light

This is an Open Access article published by World Scientific Publishing Company. It is distributed under the terms of the Creative Commons Attribution 4.0 (CC-BY) License. Further distribution of this work is permitted, provided the original work is properly cited. 


\section{Paulucci, J. E. Horvath \& O. Benvenuto}

curve indicate it may be compatible with that of a kilonova depending on the specific details of the ejecta.

Keywords: Strange Stars, Kilonova, Mergers.

PACS numbers: 26.30.-k, 97.60.Jd

\section{Introduction}

The exact composition of neutron stars is still under debate and possibilities range from proton, neutrons, and electrons to the presence of more exotic components (such as pions) and even total deconfinement to quark matter (see Ref. ${ }^{1}$ for a broad review on the subject and references therein). Recent pulsar mass measurements ${ }^{2-3}$ point to a rather stiff equation of state but no definite answer can be provided yet.

Among the possibilities, three compositions of these compact objects are widely considered: neutron stars, made of hadronic matter only, hybrid stars (with a quark core, either two - up and down - or three - up, down, and strange - flavors) and strange stars.

We investigate the nucleosynthesis and light curve that would result from a strange star - strange star (SS) merger. Given the presumed high abundance of neutrons in the matter ejected in a merger of two neutron stars (NS) or a neutron star and a black hole, r-process nucleosynthesis is expected to take place. This would render a light curve that peaks a few days following the short gamma-ray burst in the infrared region due to the high opacity of lanthanide-rich matter, a kilonova. Two such events have been observed: in 2013 (see Refs. ${ }^{4,5}$ ), with characteristics that indicate that the origin was the merger of two neutron stars and a neutron star and a black hole, respectively.

\section{Characteristics of the Ejecta}

Working within the statistical multi-fragmentation model, as presented in Paulucci \& Horvath 2014 (see Ref. ${ }^{6}$ ), we have calculated the fragmentation spectra of strange quark matter in a compact star merger scenario. The amount of ejected matter that should remain as strange quark matter (strangelets) and the amount that should decay into ordinary matter for different fragmentation temperatures indicates that no significant strangelet survival should be expected after fragmentation, independently of the fragmentation temperature and strange quark matter equation of state (with or without pairing).

Given that most of the ejected matter will be ordinary nuclear matter, the nucleosynthesis process will likely be a mass buildup from protons and neutrons. In order to evaluate the maximum mass achieved, we need to obtain the freeze out temperature and the neutron/proton ratio at this time.

Matter will be ejected with a typical speed of $0.1-0.3 \mathrm{c}$ (see Refs. ${ }^{7},{ }^{8}$ ) and will initially expand freely into the interstellar medium. This will cause the radius to 
grow from $R_{0} \approx 20 \mathrm{~km}$ linearly with time:

$$
R(t)=R_{0}+v t
$$

consequently the density, initially $\sim 4 B \sim 2 \rho_{0}$, will drop as $t^{-3}$ if one considers spherical expansion.

The ejected matter is initially at a temperature of $\sim 5-10 \mathrm{MeV}$. The temperature evolution will be

$$
\begin{aligned}
& T V^{\gamma-1}=\text { constant } \\
& T=T_{0}\left(\frac{1}{1+v / R_{0} t}\right),
\end{aligned}
$$

considering adiabatic expansion of a relativistic monoatomic ideal gas $(\gamma=4 / 3)$.

Until the freeze out temperature, protons and neutrons will be in chemical equilibrium with relative abundances given by

$$
\frac{n_{n}}{n_{p}} \sim \exp -\frac{\left(m_{n}-m_{p}\right) c^{2}}{k T},
$$

with the mass difference given by $1.29 \mathrm{MeV}$.

The equilibrium reaction rates will be roughly given by

$$
\eta \sim\left(\frac{k T}{1 M e V}\right)^{5} s^{-1}
$$

The freeze-out temperature is determined when the reaction rate drops below the expansion rate:

$$
\eta<\frac{\dot{R}}{R}=\frac{v}{R_{0}+v t} .
$$

Using equations (3) and (5), we can write this condition as

$$
k T_{\text {freezeout }}=\left(\frac{v}{R_{0} k T_{0}}\right)^{1 / 4}(M e V) .
$$

From the freeze-out temperature to $\sim 1 \mathrm{MeV}$, neutrons will decay. After $\Delta t=t(1 \mathrm{MeV})-t\left(T_{\text {freezeout }}\right)$, the final composition ratio for the beginning of the nucleosynthesis process will be given by

$$
\left.\left.\frac{n_{n}}{n_{p}}\right|_{T=1 M e V} \sim \frac{n_{n}}{n_{p}}\right|_{T_{\text {freezeout }}} \exp \left(-\frac{\Delta t}{\tau_{n}}\right),
$$

being $\tau_{n}$ the neutron mean lifetime.

Results are shown in Table 1. Since the mean neutron lifetime is much greater than $\Delta t$, the exponential argument is close to zero, which means that the final neutron to proton ratios are essentially the same as those in the freeze-out. Compared to the Big Bang nucleosyntesis, when there were 7 protons for each neutron, here we have 1.3-1.4 protons for each neutron. Typical values for the amount of ejected matter are $10^{-5}-10^{-2} M_{\odot}$ (see Refs. $\left.{ }^{7}, 8\right)$, depending on the equation of state and asymmetry of the progenitors. 


\section{Paulucci, J. E. Horvath \& O. Benvenuto}

Table 1. Freezeout temperature, radius, and proton to neutron ratio for different values of initial temperature and speed of the ejecta for a spherical expansion with initial radius of $20 \mathrm{~km}$ along with the time it takes for the temperature to drop from the freeze-out one to $1 \mathrm{MeV}$ and the corresponding proton to neutron ratio. For the case of $\mathrm{T}=5 \mathrm{MeV}$ and $\mathrm{v} / \mathrm{c}=0.3$, the freeze-out temperature is higher than the initial one. In this case, we consider the system to fragment immediately.

\begin{tabular}{ccccccc}
\hline $\mathrm{v} / \mathrm{c}$ & $\begin{array}{c}T_{\text {initial }} \\
(\mathrm{MeV})\end{array}$ & $\begin{array}{c}T_{\text {freezeout }} \\
(\mathrm{MeV})\end{array}$ & $\begin{array}{c}R_{\text {freezeout }} \\
(\mathrm{km})\end{array}$ & $\begin{array}{c}\Delta t \\
(\mathrm{~ms})\end{array}$ & $\left(n_{n} / n_{p}\right)_{\text {freezeout }}$ & $\left(n_{n} / n_{p}\right)_{\text {final }}$ \\
\hline 0.1 & 5 & 4.2 & 24.0 & 2.53 & 0.73 & 0.73 \\
0.1 & 10 & 3.5 & 57.1 & 4.76 & 0.69 & 0.69 \\
0.3 & 5 & $*$ & 20.0 & 0.89 & 0.77 & 0.77 \\
0.3 & 10 & 4.6 & 43.4 & 1.74 & 0.76 & 0.76 \\
\hline
\end{tabular}

\section{Nucleosynthesis: Numerical Results}

For the nucleosynthesis calculation we employed the TORCH code ${ }^{9}$, a general nuclear reaction network code. As expected, the obtained neutron to proton ratio renders a nucleosynthesis process which is effective in creating elements in the first mass peak. Fig. (1) give the most abundant elements created. The final mass fractions are very insensitive to the initial conditions found in the previous section given the dynamics employed.

Different radioactive elements are produced that will contribute to the light curve when decaying. Fig. (2) show the temporal evolution of the most abundant of those elements along with the energy output they produce.

Finally in Fig. (3) we present the light curve and effective temperature assuming a black-body emission as a function of time considering the expansion speed of the ejecta to be $v=0.1 c$. It indicates that the total energy output could be compatible with a kilonova event, although the details of the wavelength peak emission has still

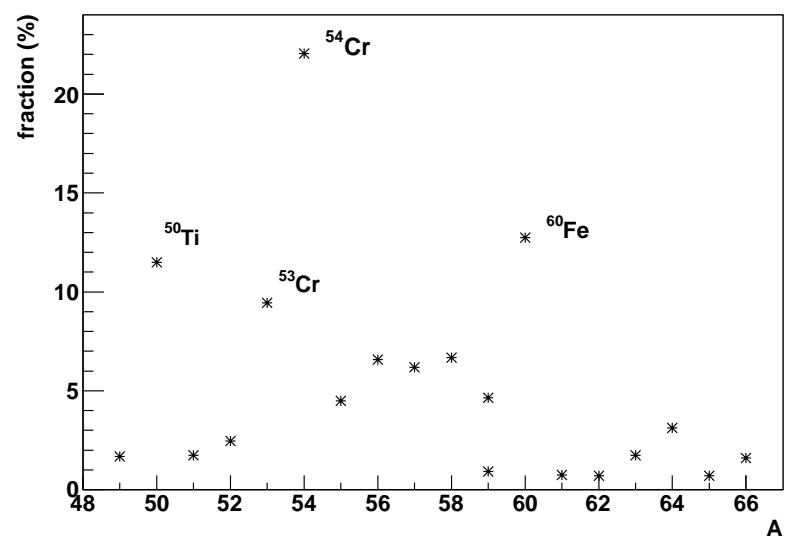

Fig. 1. Most abundant elements produced in a strange star-strange star merger. 

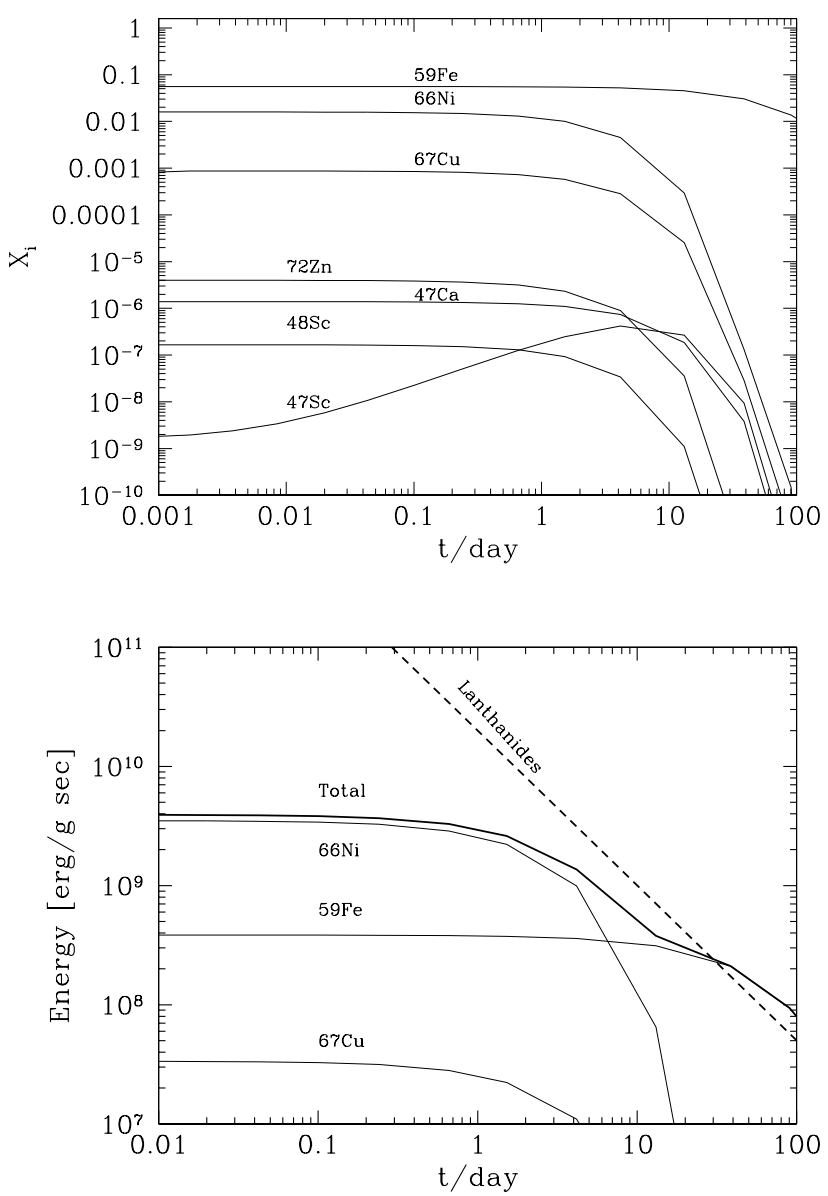

Fig. 2. The upper panel shows the abundance of the most important radioactive elements produced in a strange star-strange star merger as a function of time whereas in the lower panel the energy output from their decay in presented and compared with the one expected from lanthanide production (dashed line).

to be analyzed. Also, the influence of the expansion dynamics may be of fundamental importance.

\section{Conclusions and Perspectives}

If strange quark matter is the true ground state of cold barionic matter and is to be found inside compact stars, forming strange stars, we have shown that the nucleosynthesis following the merger of two such objects would render a very different picture from the standard scenario. In particular, the most prominent feature would be the total absence of lanthanides with a mass buildup populating the low mass 


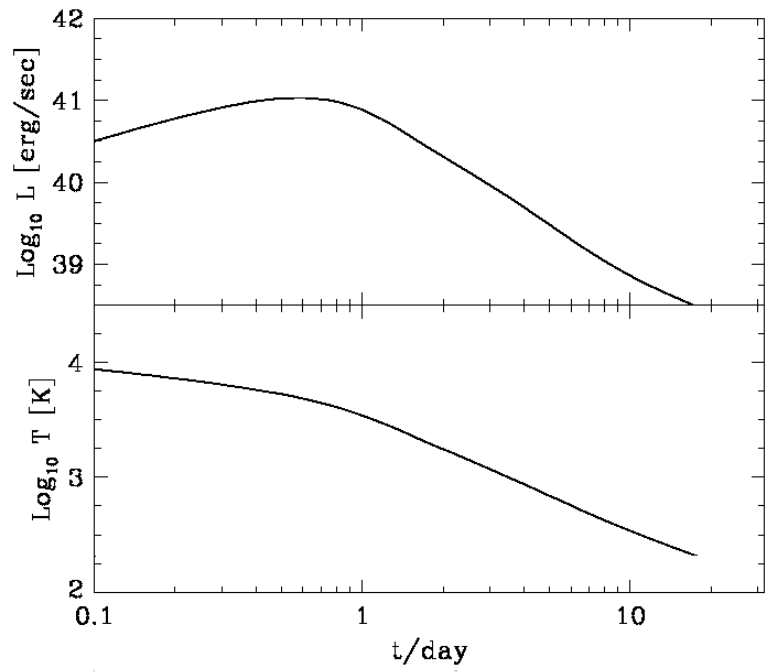

Fig. 3. Light curve (top) and effective temperature (bottom) as a function of time for a strange star-strange star merger scenario.

$(A<70)$ region. The results obtained with this simple approach are encouraging due to the production of many radioactive elements that could power the light curve. We intend to investigate the role of the ejecta dynamics on the produced elements as well as the possibility of crust elements (made of high mass elements) as seed nuclei and the reproducibility of the infrared glow at the time seen for the 2013 kilonova.

\section{Acknowledgments}

We acknowledge the financial support received from Fapesp, Fundação de Amparo à Pesquisa do Estado de São Paulo (2013/26258-4).

\section{References}

1. J. M. Lattimer, New Astron. Rev. 54, 101 (2010).

2. P. Demorest et al., Nature 467, 1081 (2010).

3. J. Antoniadis et al., Science 340, 6131 (2013).

4. N. R. Tanvir et al., Nature 500, 547 (2013); E. Berger, W. Fong, and R. Chornock, ApJ 774, L23 (2013).

5. Z.-P. Jin, X. Li, Z. Cano, S. Covino, Y.-Z. Fan, and D.-M. Wei, ApJ 811, L22 (2015).

6. L. Paulucci and J. E. Horvath, Physics Letters B 733, 164 (2014).

7. A. Bauswein et al., Phys.Rev.Lett. 103, 011101 (2009).

8. A. Bauswein, S. Goriely, H.-T. Janka, ApJ 773, 78 (2013).

9. Software available at http://cococubed.asu.edu/code_pages/net_torch.shtml. 